\title{
La actitud de los recursos humanos de las organizaciones ante la complejidad de las aplicaciones SaaS
}

\section{The attitude of human resources of organizations to the complexity of SaaS applications}

\section{A atitude dos recursos humanos das organizações perante a complexidade dos aplicativos SaaS}

\author{
Pedro R. Palos \\ Universidad de Extremadura, Cáceres, España \\ ppalos@unex.es \\ Marisol B. Correia \\ Universidade do Algarve, Faro, Portugal e CEG-IST, Universidade de Lisboa, Lisboa, Portugal \\ mcorreia@ualg.pt
}

\begin{abstract}
Resumen
El objetivo de este trabajo de investigación es contribuir al entendimiento de la adopción de la tecnología cloud y su aportación en la mejora de competitividad de una organización desde la perspectiva de los recursos humanos. Esta investigación estudia la adopción de esta tecnología en función del tamaño de la organización y de la categoría laboral del encuestado. Los resultados ponen de manifiesto la influencia de la complejidad tecnológica, el apoyo de la alta dirección, la comunicación, la formación y la actitud hacia el sistema en la intención de uso final del cloud, especialmente en función del tamaño de la organización que adopta la nube y de la categoría laboral del encuestado que pertenece a la organización.
\end{abstract}

Palabras clave: cloud computing; economía digital; adopción tecnológica; capital humano; complejidad tecnológica; intención de uso.

\begin{abstract}
The aim of this research is to contribute to a better understanding of adoption of cloud technology and its contribution in improving competitiveness of an organization from the perspective of human resources. This research studies the adoption of this technology depending on the size of organization and of the employment category of respondent. The results show the influence of technological complexity, the support of senior management, communication, training and attitude towards the system in intended end use of cloud, especially depending on the size of the organization that adopts the cloud and of the employment category of respondent belonging to the organization.
\end{abstract}

Keywords: cloud computing; digital economy; technology adoption; human capital; technological complexity; intention to use.

Dos Algarves: A Multidisciplinary e-Journal, 28 - 2016.

ISBN 2182-5580 ๑ ESGHT-University of the Algarve, Portugal.

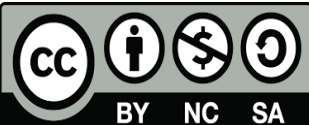

To cite this article: Palos, P.R. \& Correia, M.B. (2016). La actitud de los recursos humanos de las organizaciones ante la complejidad de las aplicaciones SaaS. Dos Algarves: A Multidisciplinary e-Journal, 28, 87-103. doi: 10.18089/DAMeJ.2016.28.6 


\section{Resumo}

O objetivo deste trabalho é contribuir para a compreensão da adoção da tecnologia cloud e a sua contribuição na melhoria da competitividade de uma organização do ponto de vista dos recursos humanos. Esta investigação estuda a adoção desta tecnologia, em função do tamanho da organização e da categoria profissional do entrevistado. Os resultados mostram a influência da complexidade tecnológica, do apoio da direção, da comunicação, da formação e da atitude em relação ao sistema na intenção de uso da cloud, em particular, em função do tamanho da organização que adota a nuvem e da categoria profissional do entrevistado que pertence à organização.

Palavras-chave: cloud computing; economia digital; adoção tecnológica; capital humano; complexidade tecnológica; intenção de usos.

\section{Introducción}

La adopción de las TIC (Tecnologías de Información y Comunicación) basadas en Internet ha hecho posible el desarrollo del paradigma de todo como servicio, donde el usuario paga sólo por el uso realizado del servicio.

Cloud o nube, es el nombre y símbolo que se usa generalmente para representar Internet. Se establece un concepto de abstracción (sistemas físicos que no se especifican, almacenamiento de datos en ubicaciones desconocidas, acceso ubicuo de los usuarios y administraciones subcontratadas).

Computing o computación, reúne los conceptos de informática, lógica de coordinación y almacenamiento. Es así como el cloud computing consiste en mover la computación del simple ordenador personal o centro de datos convencional hacia Internet. Es un cambio de paradigma real en el modo en que se utilizan los sistemas, conformando un nuevo modelo de abastecimiento de recursos para montar aplicaciones y para accesos de usuarios independientes de plataformas a los servicios.

Esta tecnología como concepto se puede llegar a explicar de dos formas. En una, la nube e Internet se confunden en un mismo concepto. De tal forma que referirse a servicios de la nube, es hacerlo a cualquier servicio ofrecido a través de Internet. También se podría llegar a entender desde el concepto más extendido, para que se usa cloud computing o computación en la nube, entendiéndolo como un conjunto de tecnologías que aportan una serie de ventajas tanto para el cliente como para el proveedor de servicios y que hacen posible verdaderas "economías de escala” en la prestación de servicios a través de Internet, reduciendo costes y aumentando la escalabilidad.

No se puede definir la nube sin sus servicios. Para ello hay que conceptualizar que son los Proveedores de Servicios de Aplicación (Application Service Provider [ASP]). Cloud parece una evolución desde este mismo concepto, visto desde la perspectiva de servicio. En este modelo se ejecuta una instancia por cada cliente del ASP, es decir, no se puede dar servicio a múltiples clientes de distintas empresas con un mismo servicio o software, algo que, como se verá más adelante, si es una característica que la tecnología cloud computing incorpora.

Para el Instituto Nacional de Estándares y Tecnología de los Estados Unidos de América (National Institute of Standards and Technology [NIST], 2011) el cloud computing es un modelo para habilitar el acceso a un conjunto de servicios computacionales (redes, servidores, almacenamiento, aplicaciones y servicios) de manera conveniente y por demanda, que pueden ser rápidamente aprovisionados y liberados con un esfuerzo administrativo y una interacción con el proveedor de servicio mínima (Forrester Research, 
2009; Heiser \& Nicolett, 2008; Buyya, Yeo, Venugopal, Broberg \& Brandic, 2009).

Es una propuesta tecnológica que permite ofrecer servicios informáticos a través de Internet en la que los recursos y el software se ofrecen bajo demanda. "El objetivo de este nuevo modelo es que la empresa o el usuario final no tengan que preocuparse por los detalles técnicos y puedan utilizar cualquier aplicación con su navegador Web" (Red.es, 2011). Se toman tecnología, servicios y aplicaciones y se convierten en utilidades de autoservicio. Los sistemas y el almacenamiento pueden abastecerse según necesidades evaluando los costes en base a unos contadores, permitiendo una escalabilidad ágil de los recursos, virtualizando sistemas, reuniendo y compartiendo recursos.

Este modelo ofrece grandes posibilidades para las organizaciones, tanto en términos de inversión como en economías de escala, deslocalización, acceso a la información desde cualquier lugar, etc.

El objetivo de este trabajo de investigación es contribuir a un mejor entendimiento de la evolución, en términos de adopción, de la tecnología cloud desde la perspectiva de los recursos humanos de la organización. Por tanto, se pretende estudiar la evolución de esta tecnología en función del tamaño de las organizaciones, especialmente en función de la dimensión de su capital humano (número de empleados) y de la categoría laboral del encuestado. Los resultados ponen de manifiesto la influencia de la complejidad tecnológica, el apoyo de la alta dirección, la comunicación, la formación y la actitud hacia el sistema en función del tamaño de la organización (número de empleados) que adopta la nube y de la categoría laboral del encuestado que responde en nombre de la organización.

Este artículo se estructura en cinco apartados. Tras esta primera parte de introducción, el segundo presenta las características asociadas al cloud computing, los principios básicos de su adopción, bien como los modelos de prestación de servicios en la nube. Posteriormente, el tercer apartado se refiere a la metodología, donde se explicita el objeto de estudio, las variables de influencia y las hipótesis planteadas, mientras que el cuarto apartado presenta un análisis en función del número de empleados de la organización y de la categoría laboral o puesto desempeñado por la persona que responde el cuestionario. Por último, el quinto apartado enuncia las conclusiones principales, sus implicaciones y posibles investigaciones futuras que se podrían llevar a cabo.

\section{Marco conceptual}

El cloud computing se articula en torno a un modelo, cuya composición se basa en cinco características principales (NIST, 2011), las cuales se pueden también conceptualizar como beneficios:

- Auto-servicio basado en la demanda: esta característica viene definida por aquellas solicitudes de servicio que pida el usuario a través de Internet. Los servicios son facturados exclusivamente por el factor tiempo de uso. En esta característica o beneficio el cliente o el usuario no necesitan interactuar directamente con técnicos;

- Acceso omnipresente: el conjunto de recursos y servicios que ofrece el cloud computing está a disposición de los usuarios. Su carácter ubicuo u omnipresente estriba en que es multiplataforma y se puede acceder desde cualquier medio propio de la red. Esto quiere decir que existe una verdadera independencia del medio con multitud de clientes de carácter dispar o perfiles diferentes; 
- Repositorio comunitario de recursos o propiedad múltiple de recursos: el modelo cloud se caracteriza por tanto por ser un conjunto de recursos disperso y replicado por criterios de accesibilidad y proximidad de manera internacional, el cual es multialquiler a multitud de clientes que lo comparten de forma comunitaria. Esto es conocido por el concepto de independencia del sitio o lugar que aprovecha el carácter de asignación dinámica de servicios y recursos, ya sean físicos o virtuales, en función de las necesidades de los usuarios;

- Elasticidad dinámica: los servicios ofrecidos en cloud, su calidad y cantidad disminuirán o aumentarán de forma veloz en función de las necesidades mostradas por los usuarios en tiempo real. Esto es de forma dinámica y elástica a la vez. Se podrán producir asignaciones en el sistema de recursos escalando sistemas (es decir hacia una computación más potente) o ajustando el número de sistemas (hacia un mayor número de procesadores en paralelo). Las asignaciones serán definidas de manera automatizada o seguida por un técnico;

- Carácter ponderable del servicio: esta característica le confiere a los servicios cloud, un atributo propio de las llamadas "commodities", es decir de los mercados de materias primas o productos básicos, los cuales cuentan con un carácter descentralizado en los que se negocian estos productos no manufacturados y genéricos con bajo nivel de diferenciación. Esta comparación se basa en el carácter ponderable o que de medición tiene la cantidad de almacenamiento, el número de transacciones, el ancho de banda, la memoria o el número de núcleos. Estos factores medibles son la base de la facturación o el pago que por su tiempo de uso y cantidad, abona el cliente, sin grandes diferenciaciones a priori.

\subsection{Características complementarias}

A estas cinco características definidas por el NIST (2011), podemos añadir otras (Sosinski, 2011):

- Costes más bajos: puesto que los servicios en la nube operan con una eficacia más alta y por tanto con una utilización mayor, se origina una reducción significativa de los costes debido a la ley de la oferta y la demanda;

- Facilidad de utilización: no se necesitan licencias de software o hardware para implementar el servicio;

- Calidad de servicio (Quality of Service [QoS]): será algo fijado en el contrato con el proveedor (acuerdos de niveles de servicios);

- Fiabilidad: la capacidad para proporcionar equilibrio de carga y conmutación tras error hace que el sistema sea muy fiable;

- Administración TIC subcontratada: administración externa de los servicios TIC, mientras que la empresa se centra en administrar sus negocios;

- Mantenimiento y actualización simplificados: al estar los sistemas centralizados, las actualizaciones de los mismos se realizan de una forma más sencilla. Supone un acceso sencillo a las versiones más recientes;

- Barreras de entrada baja: concepto vinculado al desembolso de capital alineado con el crecimiento y definido como coste variable. 


\subsection{Cloud y empresa: Principios básicos de adopción}

La tecnología cloud supone un avance, pero su adopción debe hacerse bajo un esquema modular y que ofrezca grandes facilidades de reconfiguración, en el que se puede flexiblemente acumular recursos o servicios que satisfagan las cambiantes exigencias del mercado.

No obstante, el concepto aún se encuentra en España en particular y globalmente en general en su etapa más incipiente de adopción afectando de forma dispar a los dos sectores clave del tejido empresarial: la gran empresa y la Pyme.

Frost y Sullivan (2008) identificaron unos principios básicos para la adopción de la nube en cada una de estas dos categorías en que se divide el tejido empresarial:

- Asumir claramente, por parte de la alta dirección, que el concepto que supone esta nueva filosofía de uso de recursos, servicios y aplicaciones, así como sus beneficios es una de las claves o principios. Unido al principio anterior estaría desplegar en un tiempo determinado esta tecnología de arriba hacia abajo, lo que significa que de no hacerlo se corre el riesgo de generar mucha controversia y dificultades en la organización que conllevaran mayores costes en formación de los usuarios, que no observan en sus superiores verdadera sensibilidad hacia este nuevo modelo tecnológico;

- Estandarizar en un diagrama de procesos basados en el cloud computing, con definiciones claras y precisas de cada uno de ellos para mejorar el proceso de adopción y uso de esta nueva tecnología;

- Implementar un programa formativo y nombrar responsables del proyecto de adopción. Con ello se conseguirá una mejor dirección del proceso al estar unificado;

- Observar otros despliegues y adopciones de esta tecnología, llevando a cabo un aprendizaje de buenas prácticas que nos permita conseguir esos mismos éxitos;

- Llevar a cabo un análisis real de la situación de partida, observando especialmente la tecnología utilizada actualmente, su infraestructura actual frente al hecho de que las tecnologías cloud poseen una importante propiedad: la escalabilidad que es vital para el crecimiento de una organización;

- Implementar o adoptar esta nueva tecnología de forma gradual, llevando a cabo la transición de forma escalonada, de menor a mayor importancia, es decir de menor a mayor criticidad del proceso. A partir de ahí y eligiendo este punto de partida, habría que ir viendo los beneficios en un diagrama temporal.

Mientras que los servicios de la nube, como Webmail, Flickr o YouTube han sido ampliamente utilizados por los individuos durante algún tiempo, no es hasta hace relativamente poco tiempo, menos de dos décadas en realidad, cuando las organizaciones han comenzado a utilizar los servicios de la nube como una herramienta para el cumplimiento de sus necesidades TIC.

Para que la computación en la nube pueda crecer, es importante comprender los factores que pueden influir en su tasa de adopción por parte de las organizaciones, especialmente empresas. Es de destacar el estudio de Lin y Chen (2012), que tiene como objetivo investigar cómo el cloud computing es percibido por los profesionales TIC y las preocupaciones que los profesionales TIC tienen en lo que respecta a la adopción de 
servicios cloud. Este estudio se realizó en Taiwán en 2009, cuando la computación en la nube era todavía un nuevo fenómeno para la mayoría de las organizaciones y ha constituido una excelente oportunidad para estudiar cómo se percibe una innovación y qué factores pueden fomentar y evitar su aplicación anticipada.

El estudio recabó las opiniones de los profesionales TIC ya que estos son más propensos a experimentar, adoptar, o dicho de otra manera, a promover el uso de la computación en la nube. Demostrando que la teoría de la difusión de la innovación es la base teórica utilizada para apoyar la identificación de la factores que alientan y prevén su adopción (Rogers, 1995).

\subsection{Modelos de prestación de servicios en la nube}

Los modelos existentes se pueden clasificar en un primer criterio basado en la forma de prestar los servicios. Este criterio lo divide en tres tipos:

- Software-as-a-Service (SaaS);

- Platform-as-a-Service (PaaS);

- Infraestructure-as-a-Service (laaS).

Cada uno de los tipos de servicio tiene objetivos diferentes y se dirigen a distintos clientes; sin embargo comparten un modelo de negocio común en el que se alquila el uso de los recursos de computación, incluyendo servicios, aplicaciones, infraestructuras y plataformas para los clientes.

Este modelo es similar al modelo de ASP en el que un proveedor de servicios proporciona el software, la infraestructura, personas y el mantenimiento para ejecutar de forma personalizada de cara al cliente (Wang, Chow, Wang, Ren \& Lou, 2013).

Así, los modelos de SaaS y de servicios pueden ser entendidos como una variación de ASP, donde los clientes pagan, alquilan o se suscriben a aplicaciones o servicios de los proveedores de la nube, como podrían ser el almacenamiento, acceso a base de datos o a distintas capacidades, siempre a través de Internet (Leavitt, 2009).

Ambos, SaaS y el resto de modelos de servicios, tienen como objetivo proporcionar un funcionamiento libre de problemas para los usuarios finales y permiten a los clientes corporativos verse libres de administrar sus recursos TIC (Pearlson \& Saunders, 2009).

Por lo tanto, se puede decir que el SaaS ha emergido como una innovadora forma de acercar y ofrecer al usuario aplicaciones de software basado en cloud computing (Chou \& Chou, 2007). En este modelo, los proveedores de SaaS despliegan aplicaciones de software en los servidores de la nube para que los usuarios soliciten en función de sus necesidades y paguen por los servicios de acuerdo con su uso (Armbrust et al., 2010).

Además, SaaS mejora la calidad de servicios del software a través de la actualización de la aplicación y los datos de copia de seguridad automática (Xin \& Levina, 2008) y por tanto confianza del cliente (Goode, Lin, Tsai \& Jiang, 2015).

El SaaS es definido como un modelo de distribución de software, pero también es considerado como un servicio al que se accede, a través de Internet, mediante un navegador web, bajo la demanda del propio usuario (Observatorio Nacional de las Telecomunicaciones y de la Sociedad de la Información [ONTSI], 2012).

La computación en la nube ha ido más allá del básico SaaS y la prestación de servicios y proporciona laaS y PaaS. PaaS proporciona un entorno de desarrollo de la aplicación, total o 
parcial, que permite a los desarrolladores acceder a los recursos para el desarrollo de aplicaciones y colaborar con otras personas en línea (Mathur \& Nishchal, 2010). La solución de Amazon de almacenamiento simple (S3) y Microsoft Azure plataforma de Servicios son las soluciones más conocidas de PaaS.

IaaS ofrece a los clientes un amplio conjunto de recursos informáticos, por ejemplo máquinas virtuales para ofrecer una infraestructura de computación a los clientes a través de Internet. laaS está dirigido a las empresas TIC y a desarrolladores de software para permitirles aumentar o disminuir el número de máquinas virtuales funcionando, en función de su carga de trabajo para promover la eficiencia en el uso de los recursos TIC, los ejemplos más mencionados suelen ser Elastic Compute de Amazon Cloud (EC2) y Mosso Hosting Cloud.

Dicho lo anterior resulta evidente que el cloud computing desafía la comprensión actual de recursos TIC. Los recursos TIC ya no se consideran como unos productos más, sino que se entienden como los servicios que se pueden alquilar y suscribir mediante contrato con los proveedores y con el denominador común de que siempre se accede a través de Internet.

Es por lo tanto, un argumento contrastado que la computación en la nube representa un cambio de paradigma de la computación y la gestión de los servicios de las TIC, tanto para la virtualización de recursos TIC, como su gestión (Armbrust et al., 2010; Buyya et al., 2009).

\section{Metodología}

\subsection{Objeto de estudio}

Las 615 empresas que componen nuestro universo objeto de estudio han sido conseguidas entrando en contacto con organizaciones empresariales del sector. En todos los casos, el perfil buscado es el de empresas que usan el cloud computing como componente estratégico en Andalucía.

Una vez confeccionado el censo de empresas y organizaciones que forman parte de la población objeto de estudio, se alcanzó una población a la cual se contactó en su totalidad. De este censo, 161 empresas cumplimentaron el cuestionario, obteniéndose una cifra final de 150 válidos.

La técnica cuantitativa de investigación que se ha llevado a cabo ha sido la encuesta, materializada en la encuesta online, aunque reforzada con supervisión telefónica, entre los meses de septiembre y noviembre de 2015. Para ello las preguntas se presentaron en un sitio web cuyo enlace fue enviado a organizaciones que constituyen la población objeto de estudio y se utilizó una escala de Likert de cinco niveles, donde el 1 ha sido: "Totalmente en desacuerdo" y el 5 fue: "Totalmente de acuerdo". Se ha llevado a cabo una prueba paramétrica, ya que se conoce el censo y por tanto las condiciones previas en la población objeto de estudio. La distribución es normal y existe homogeneidad de varianzas.

Con independencia del cargo desempeñado, en todos los casos el cuestionario fue respondido por la persona con mayor conocimiento del proceso de adopción del cloud dentro de la organización o directamente por la dirección.

\subsection{Variables de influencia}

Se han estudiado las variables clasificatorias siguientes: número de empleados de las organizaciones y categoría laboral o puesto desempeñado del representante de la organización que respondió a la encuesta. Las preguntas o ítems de las encuestas se han 
clasificado en seis grupos (ver Tabla 1).

Tabla 1. Constructos e ítems empleados

CONSTRUCTO

VARIABLE

(AHS1) El sistema cloud computing me proveerá acceso a la mayoría de los datos.

ACTITUDES HACIA EL (AHS2) El sistema cloud computing será/es mejor que la anterior Tecnología SISTEMA de la Información.

(AHS3) El sistema cloud computing proveerá información exacta.

(AHS4) El sistema cloud computing proveerá información integrada, oportuna y fiable.

(IU1) Espero usar el sistema cloud computing.

INTENCION DE USO (IU2) Espero que la información del nuevo sistema de cloud computing sea útil.

(AAD1) La alta dirección está interesada en el sistema de cloud computing. (AAD2) La alta dirección comprende la importancia del sistema de cloud computing.

\section{APOYO ALTA \\ DIRECCIÓN}

COMPLEJIDAD

TECNOLÓGICA

COMUNICACIÓN

FORMACIÓN DEL PERSONAL

(AAD3) La alta dirección apoya al sistema de cloud computing.

(AAD4) La alta dirección comprende las oportunidades del sistema de cloud computing.

(CT1) Es difícil comprender lo que hace el sistema de cloud computing.

(CT2) Usar el sistema de cloud computing me ocupa demasiado tiempo.

(CT3) Necesito mucho esfuerzo para aprender a usar el sistema cloud computing.

(CT4) En general, el sistema de cloud computing es muy complejo de usar.

(CO1) Existe una comunicación fluida con respecto al sistema de cloud computing.

( $\left.\mathrm{CO}_{2}\right)$ No existen obstáculos para tratar el tema del cloud computing.

(CO3) Considero que la información que recibo del sistema de cloud computing es sincera.

(FP1) La formación recibida fue completa.

$(\mathrm{FP} 2)$ La formación recibida me proporciona confianza en el sistema de cloud computing.

(FP3) La formación fue adecuadamente extensa y detallada.

(FP4) Los formadores eran conocedores del sistema de cloud computing y me permitían conocerlo.

Fuente: Elaboración propia.

- Apoyo de la Alta Dirección (AAD): el interés de la alta dirección en la implantación, se puede traducir en una realización adecuada de la selección del sistema, la planificación del proceso, la formulación de políticas, la elección de expertos, la coordinación, la asignación de recursos y de la arquitectura, el apoyo a la reingeniería de procesos, la evaluación del rendimiento o la gestión de las expectativas (Shah \& Siddiqui, 2006);

- Comunicación (CO): el proceso de transferencia de información entre los miembros de un equipo de trabajo u organización (Hsu, Shih, Chiang \& Liu, 2012);

- Formación del personal (FP): se describe como el grado en el que una empresa instruye a sus empleados en el uso de una herramienta en términos de calidad y cantidad (Schillewaert, Ahearne, Frambach \& Moenaert, 2005); 
- Complejidad tecnológica (CT): el grado a través del cual una innovación es percibida como relativamente difícil de comprender y usar (Rogers, 2003) de aspectos como el grado de dificultad de las habilidades necesarias para utilizar las tecnologías entre los empleados (Premkumar \& Roberts, 1999);

- $\quad$ Actitud hacia el Sistema (AHS): refleja sentimientos favorables o desfavorables respecto al empleo de una determinada tecnología (Taylor \& Todd, 1995);

- Intención de Uso (IU): es el grado de comportamiento previo que se tiene a la hora de usar dicha tecnología (Taylor \& Todd, 1995).

\subsection{Hipótesis}

Las hipótesis formuladas han sido planteadas como se expone en la Tabla 2.

Estas hipótesis se contrastarán a través de un análisis de muestras independientes a partir de las pruebas $t$ de Student o ANOVA de un factor. Asimismo, se han incluido en este análisis los indicadores de otras variables del modelo que se consideran especialmente relacionadas con el puesto desempeñado por la persona que responde el cuestionario y el tamaño de la organización (número de empleados).

Tabla 2. Hipótesis planteadas

\begin{tabular}{c|c}
\hline $\mathbf{N}^{{ }^{\circ}}$ & Hipótesis \\
\hline $\mathbf{1}$ & El número de empleados de la organización influye en la actitud hacia el \\
sistema \\
$\mathbf{3}$ & El número de empleados de la organización influye en la intención de uso \\
$\mathbf{4}$ & La categoría laboral influye en la intención de uso \\
$\mathbf{5}$ & El número de empleados de la organización influye en la complejidad \\
6 & La categoría laboral influye en la complejidad tecnológica \\
7 & La categoría laboral influye en la formación profesional \\
\hline
\end{tabular}

Fuente: Elaboración propia. 


\section{Resultados}

4.1 Análisis por número de empleados

Tabla 3. Número trabajadores: medias y desviaciones típicas

\begin{tabular}{|c|c|c|c|c|c|c|c|c|c|c|c|}
\hline \multicolumn{2}{|l|}{ NTR } & AHS1 & AHS2 & $\mathrm{AHS}_{3}$ & AHS4 & IU1 & IU2 & CT1 & $\mathrm{CT} 2$ & $\mathrm{CT}_{3}$ & $\mathrm{CT}_{4}$ \\
\hline \multirow{2}{*}{$\begin{array}{c}<10 \mathrm{~T} \\
(\mathrm{~N}=76)\end{array}$} & $\bar{x}$ & 4,67 & 4,62 & 4,50 & 4,57 & 4,79 & 4,71 & 2,34 & 2,04 & 2,00 & 2,04 \\
\hline & $\Sigma$ & 0,641 & 0,632 & 0,792 & 0,660 & 0,573 & 0,607 & 1,381 & 1,194 & 1,222 & 1,125 \\
\hline \multirow{2}{*}{$\begin{array}{c}\text { De } 10 \text { a } 25 \mathrm{~T} \\
(\mathrm{~N}=36)\end{array}$} & $\bar{x}$ & 4,78 & 4,75 & 4,58 & 4,67 & 4,83 & 4,81 & 1,78 & 1,64 & 1,64 & 1,53 \\
\hline & $\Sigma$ & 0,485 & 0,554 & 0,841 & 0,632 & 0,447 & 0,401 & 0,898 & 0,798 & 1,018 & 0,609 \\
\hline \multirow{2}{*}{$\begin{array}{c}\text { De } 25 \text { a } 100 \mathrm{~T} \\
(\mathrm{~N}=23)\end{array}$} & $\bar{x}$ & 4,17 & 4,35 & 4,35 & 4,39 & 4,78 & 4,61 & 2,39 & 1,70 & 1,65 & 1,74 \\
\hline & $\Sigma$ & 1,114 & 0,832 & 0,832 & 0,783 & 0,422 & 0,656 & 1,234 & 1,063 & 0,982 & 0,964 \\
\hline \multirow{2}{*}{$\begin{array}{c}\text { De } 100 \text { a } 250 \mathrm{~T} \\
(\mathrm{~N}=3)\end{array}$} & $\bar{x}$ & 4,33 & 4,33 & 4,67 & 4,33 & 4,33 & 4,67 & 3,33 & 3,00 & 3,00 & 3,00 \\
\hline & $\sigma$ & 1,155 & 0,577 & 0,577 & 0,577 & 0,577 & 0,577 & 1,155 & 2,000 & 2,000 & 2,000 \\
\hline \multirow{2}{*}{$\begin{array}{l}>250 \mathrm{~T} \\
(\mathrm{~N}=12)\end{array}$} & $\bar{x}$ & 4,50 & 4,17 & 3,92 & 4,17 & 4,75 & 4,58 & 3,25 & 2,08 & 2,50 & 2,08 \\
\hline & 0 & 0,674 & 0,835 & 1,084 & 0,835 & 0,452 & 0,515 & 1,138 & 0,996 & 0,905 & 0,900 \\
\hline \multirow{2}{*}{$\begin{array}{c}\text { Total } \\
(\mathrm{N}=150)\end{array}$} & $\bar{x}$ & 4,60 & 4,57 & 4,45 & 4,53 & 4,79 & 4,71 & 2,31 & 1,91 & 1,92 & 1,89 \\
\hline & $\sigma$ & 0,733 & 0,680 & 0,840 & 0,692 & 0,513 & 0,562 & 1,285 & 1,105 & 1,156 & 1,024 \\
\hline
\end{tabular}

Fuente: Elaboración propia.

\subsection{Análisis por puesto desempeñado o categoría laboral}

En primer lugar, se evaluará si el cargo que ocupa en la organización la persona que respondió al cuestionario tiene alguna influencia en las variables estudiadas. Para ello, se ha dividido la muestra en dos submuestras, que están diferenciadas en función de si el encuestado es directivo $\left(n_{1}=79\right)$ o no directivo de la empresa $\left(n_{2}=71\right)$ y se estudiarán si se obtienen medias iguales o diferentes significativamente.

La Tabla 4 recoge las medias y desviaciones típicas obtenidas por ambas submuestras en los ítems que guardan más relación con el área de Recursos Humanos (Apoyo de la Alta Dirección, Comunicación, Complejidad Tecnológica y Formación del Personal), los cuales podrían estar más influidos por el cargo que ocupa la persona dentro de la organización (directivo o no directivo).

Antes de aplicar la prueba $t$ de Student, que permite determinar si existen diferencias estadísticamente significativas al comparar los promedios de las dos submuestras independientes, es necesario realizar el Test de Levene para comprobar si las varianzas son o no diferentes. Se dice que un modelo predictivo presenta homocedasticidad cuando la varianza del error de la variable endógena se mantiene a lo largo de las observaciones. Por tanto para llevar a cabo el test de hipótesis que conduce a la prueba de $t$, necesitamos ver si se cumple esa condición previamente. 
Tabla 4. Relación muestras directivos y no directivos

\begin{tabular}{|c|c|c|c|c|}
\hline Item & $\begin{array}{c}\text { Puesto de } \\
\text { trabajo }\end{array}$ & Media & Desviación típica & $\begin{array}{l}\text { Error } \\
\text { típico }\end{array}$ \\
\hline \multirow{2}{*}{ AAD1 } & No directivo & 4,34 & 1,055 & 0,125 \\
\hline & Directivo & 4,43 & 0,796 & 0,090 \\
\hline \multirow{2}{*}{ AAD2 } & No directivo & 4,34 & 1,082 & 0,128 \\
\hline & Directivo & 4,43 & 0,887 & 0,100 \\
\hline \multirow{2}{*}{$A A D_{3}$} & No directivo & 4,32 & 1,066 & 0,127 \\
\hline & Directivo & 4,37 & 0,922 & 0,104 \\
\hline \multirow{2}{*}{ AAD4 } & No directivo & 4,38 & 1,019 & 0,121 \\
\hline & Directivo & 4,22 & 1,021 & 0,115 \\
\hline \multirow{2}{*}{$\mathrm{CO} 1$} & No directivo & 2,00 & 1,134 & 0,135 \\
\hline & Directivo & 2,58 & 1,355 & 0,152 \\
\hline \multirow{2}{*}{$\mathrm{CO}_{2}$} & No directivo & 1,62 & 0,962 & 0,114 \\
\hline & Directivo & 2,19 & 1,251 & 0,141 \\
\hline \multirow{2}{*}{$\mathrm{CO}_{3}$} & No directivo & 4,07 & 1,138 & 0,135 \\
\hline & Directivo & 3,92 & 0,931 & 0,105 \\
\hline \multirow{2}{*}{ CT1 } & No directivo & 2,00 & 1,134 & 0,135 \\
\hline & Directivo & 2,58 & 1,355 & 0,152 \\
\hline \multirow{2}{*}{$\mathrm{CT} 2$} & No directivo & 1,61 & 0,870 & 0,103 \\
\hline & Directivo & 2,19 & 1,220 & 0,137 \\
\hline \multirow{2}{*}{$\mathrm{CT}_{3}$} & No directivo & 1,62 & 0,962 & 0,114 \\
\hline & Directivo & 2,19 & 1,251 & 0,141 \\
\hline \multirow{2}{*}{ CT4 } & No directivo & 1,63 & 0,832 & 0,099 \\
\hline & Directivo & 2,13 & 1,125 & 0,127 \\
\hline \multirow{2}{*}{ FP1 } & No directivo & 3,87 & 1,444 & 0,171 \\
\hline & Directivo & 3,72 & 1,320 & 0,148 \\
\hline \multirow{2}{*}{ FP2 } & No directivo & 3,92 & 1,350 & 0,160 \\
\hline & Directivo & 3,86 & 1,238 & 0,139 \\
\hline \multirow{2}{*}{$\mathrm{FP}_{3}$} & No directivo & 4,69 & 0,689 & 0,082 \\
\hline & Directivo & 4,33 & 1,034 & 0,116 \\
\hline \multirow{2}{*}{ FP4 } & No directivo & 3,90 & 1,244 & 0,148 \\
\hline & Directivo & 3,85 & 1,220 & 0,137 \\
\hline \multirow{2}{*}{$I_{1}$} & No directivo & 4,83 & 0,414 & 0,049 \\
\hline & Directivo & 4,75 & 0,588 & 0,066 \\
\hline \multirow{2}{*}{ IU2 } & No directivo & 4,79 & 0,445 & 0,053 \\
\hline & Directivo & 4,63 & 0,644 & 0,072 \\
\hline
\end{tabular}

Fuente: Elaboración propia. 
Tabla 5. Prueba de t para el cargo en la organización (I)

\begin{tabular}{|llccccc|}
\hline \multicolumn{1}{|c}{ Prueba de t o de igualdad de medias } & \multicolumn{2}{c|}{ Test de Levene } & & & \\
\cline { 2 - 5 } & & $\mathbf{F}$ & Sig. & $\mathbf{t}$ & $\begin{array}{c}\text { Sign. } \\
\text { bilateral }\end{array}$ & $\begin{array}{c}\text { Difer. de } \\
\text { medias }\end{array}$ \\
\hline AAD1 Se han asumido varianzas iguales & 2,610 & 0,108 & $-0,609$ & 0,543 & $-0,092$ \\
\hline AAD2 Se han asumido varianzas iguales & 0,759 & 0,385 & $-0,574$ & 0,567 & $-0,092$ \\
\hline AAD3 Se han asumido varianzas iguales & 0,777 & 0,379 & $-0,266$ & 0,791 & $-0,043$ \\
\hline AAD4 Se han asumido varianzas iguales & 0,240 & 0,625 & 0,989 & 0,324 & 0,165 \\
\hline
\end{tabular}

Fuente: Elaboración propia.

Tabla 6. Prueba de t para el cargo en la organización (II)

\begin{tabular}{|c|c|c|c|c|c|}
\hline \multirow[t]{2}{*}{ Prueba de t o de igualdad de medias } & \multicolumn{2}{|c|}{ Test de Levene } & \multirow[b]{2}{*}{$t$} & \multirow[b]{2}{*}{$\begin{array}{l}\text { Sign. } \\
\text { bilateral }\end{array}$} & \multirow[b]{2}{*}{$\begin{array}{c}\text { Difer.d } \\
\text { e } \\
\text { medias }\end{array}$} \\
\hline & $\mathbf{F}$ & Sig. & & & \\
\hline $\begin{array}{l}\text { CT1 No se han asumido varianzas iguales } \\
\text { Las medias son distintas }\end{array}$ & 6,588 & 0,011 & $\begin{array}{c}- \\
2,86 \\
3\end{array}$ & 0,005 & $-0,582$ \\
\hline $\begin{array}{l}\text { CT2 No se han asumido varianzas iguales } \\
\text { Las medias son distintas }\end{array}$ & 5,997 & 0,016 & $\begin{array}{c}- \\
3,401 \\
\end{array}$ & 0,001 & $-0,584$ \\
\hline $\begin{array}{l}\text { CT3 No se han asumido varianzas iguales } \\
\text { Las medias son distintas }\end{array}$ & 4,994 & 0,027 & $\begin{array}{c}- \\
3,146\end{array}$ & 0,002 & $-0,570$ \\
\hline $\begin{array}{l}\text { CT4 Se han asumido varianzas iguales } \\
\text { Las medias son distintas }\end{array}$ & 2,489 & 0,117 & $\begin{array}{c}- \\
3,06 \\
9\end{array}$ & 0,003 & $-0,493$ \\
\hline CO1 Se han asumido varianzas iguales & 1,713 & 0,193 & $\begin{array}{c}0,86 \\
6\end{array}$ & 0,388 & 0,146 \\
\hline $\begin{array}{l}\text { CO2 Se han asumido varianzas iguales } \\
\text { Las medias son distintas }\end{array}$ & 0,055 & 0,815 & 2,206 & 0,029 & 0,376 \\
\hline $\mathrm{CO}_{3}$ Se han asumido varianzas iguales & 1,534 & 0,217 & 0,173 & 0,863 & $-0,028$ \\
\hline FP1 Se han asumido varianzas iguales & 0,550 & 0,459 & $\begin{array}{c}0,26 \\
5\end{array}$ & 0,792 & 0,053 \\
\hline FP2 Se han asumido varianzas iguales & 1,236 & 0,268 & $\begin{array}{c}- \\
0,69 \\
7\end{array}$ & 0,487 & $-0,127$ \\
\hline FP3 Se han asumido varianzas iguales & 1,060 & 0,305 & 0,781 & 0,436 & $-0,151$ \\
\hline FP4 Se han asumido varianzas iguales & 1,278 & 0,260 & $-1,104$ & 0,271 & $-0,205$ \\
\hline
\end{tabular}

Fuente: Elaboración propia. 
Tabla 7. Prueba de t para el cargo en la organización (III)

\begin{tabular}{|lcccccc|}
\hline \multicolumn{1}{|c}{ Prueba de t o de igualdad de medias } & \multicolumn{2}{c|}{ Test de Levene } & & & \\
\cline { 2 - 6 } & & F & Sig. & t & $\begin{array}{c}\text { Sign. } \\
\text { bilater } \\
\text { al }\end{array}$ & $\begin{array}{c}\text { Difer. de } \\
\text { medias }\end{array}$ \\
\hline IU1 Se han asumido varianzas iguales & 3,346 & 0,069 & 1,004 & 0,317 & 0,084 \\
\hline $\begin{array}{l}\text { IU2 No se han asumido varianzas } \\
\text { iguales }\end{array}$ & 10,935 & 0,001 & 1,738 & 0,084 & 0,156 \\
\hline
\end{tabular}

Fuente: Elaboración propia.

Las Tablas 5, 6 y 7 recogen los resultados del Test de Levene y de la prueba $t$ de Student. El Test de Levene contrasta la hipótesis nula de que las varianzas son iguales. Por tanto, si la significación que acompaña al valor del estadístico $\mathrm{F}$ es menor que 0,05 rechazamos dicha hipótesis nula y aceptamos que las varianzas son distintas. En el caso de la prueba $t$, se plantea la hipótesis nula de que las medias son iguales. Por tanto, si la significación bilateral es menor que 0,05, aceptamos la hipótesis alternativa y la diferencia en las medias de ambas submuestras.

Como se puede comprobar, en la mayoría de los casos planteados no se producen diferencias estadísticamente significativas entre directivos y no directivos, con excepción de los ítems señalados (CT1: "Es difícil comprender lo que hace el sistema cloud computing"; CT2: "Usar el sistema de cloud computing me ocupa demasiado tiempo"; CT3: "Necesito mucho esfuerzo para aprender a usar el sistema cloud computing"; CT4: "En general, el sistema de cloud computing es muy complejo de usar"; $\mathrm{CO} 2:$ "No existen obstáculos para tratar el tema del cloud computing").

En primer lugar, en el caso de los ítems relacionados con la complejidad tecnológica los no directivos muestran promedios más bajos, en tanto que en el de intención de uso son los directivos los que presentan una media inferior. Las valoraciones de los no directivos muestran una menor percepción de complejidad sobre los sistemas cloud que las de los directivos, para los cuales usar el cloud supone más tiempo y esfuerzo de aprendizaje y una mayor complejidad de uso. En segundo lugar, conviene destacar que no existen diferencias entre directivos y no directivos en lo que respecta a la Intención de uso de los sistemas cloud. Por lo que respecta a los ítems de Comunicación, en general, los directivos obtienen una media más alta, es decir, valoran más positivamente la importancia de la comunicación interna en la implantación del cloud computing. En cualquier caso, esta diferencia sólo llega a ser estadísticamente significativa en el mencionado indicador $\mathrm{CO}_{2}$. 
Tabla 8. Anova de un factor: número de trabajadores

\begin{tabular}{|c|c|c|c|c|c|c|c|c|}
\hline \multirow[t]{2}{*}{$\begin{array}{c}\text { Ítems / } \\
\text { Variables } \\
\text { depend. }\end{array}$} & \multirow[t]{2}{*}{ ANOVA } & \multirow[t]{2}{*}{$\begin{array}{l}\text { Test de } \\
\text { Levene }\end{array}$} & \multicolumn{2}{|c|}{$\begin{array}{l}\text { Comparaciones } \\
\text { con diferencias } \\
\text { significativas }\end{array}$} & \multirow[t]{2}{*}{$\begin{array}{l}\text { Dif. de } \\
\text { medias } \\
(I-J)\end{array}$} & \multicolumn{3}{|c|}{ Post Hoc } \\
\hline & & & & (J) & & Test & $\begin{array}{c}\text { Error } \\
\text { Estándar }\end{array}$ & Sig. \\
\hline AHS1 & $\begin{array}{c}F=2,955 \\
\text { Sig. }=\mathbf{0 , 0 2 2}\end{array}$ & $\begin{array}{c}F=5,471 \\
\text { Sig. }=0,000\end{array}$ & - & - & - & $\begin{array}{l}\text { Games- } \\
\text { Howell }\end{array}$ & - & - \\
\hline AHS2 & $\begin{array}{c}F=2,595 \\
\text { Sig. }=0,039\end{array}$ & $\begin{array}{c}F=3,685 \\
\text { Sig. }=0,007\end{array}$ & - & - & - & $\begin{array}{l}\text { Games- } \\
\text { Howell }\end{array}$ & - & - \\
\hline AHS3 & $\begin{array}{c}F=1,667 \\
\text { Sig. }=0,161\end{array}$ & $\begin{array}{c}F=1,391 \\
\text { Sig. }=0,240\end{array}$ & - & - & - & - & - & - \\
\hline AHS4 & $\begin{array}{c}F=1,541 \\
\text { Sig. }=0,193\end{array}$ & $\begin{array}{c}F=1,493 \\
\text { Sig. }=0,207\end{array}$ & - & - & - & - & - & - \\
\hline CT1 & $\begin{array}{c}F=3,952 \\
\text { Sig. }=\mathbf{0 , 0 0 4}\end{array}$ & $\begin{array}{c}F=3,155 \\
\text { Sig. }=0,016\end{array}$ & $\begin{array}{c}>250 \\
T\end{array}$ & $\begin{array}{c}\text { De } 10 \mathrm{a} \\
25 \mathrm{~T}\end{array}$ & 1,472 & $\begin{array}{l}\text { Games- } \\
\text { Howell }\end{array}$ & 0,361 & 0,007 \\
\hline CT2 & $\begin{array}{c}F=1,865 \\
\text { Sig. }=0,120\end{array}$ & $\begin{array}{c}F=1,122 \\
\text { Sig. }=0,349\end{array}$ & - & - & - & - & - & - \\
\hline $\mathrm{CT}_{3}$ & $\begin{array}{c}F=2,433 \\
\text { Sig. }=0,050\end{array}$ & $\begin{array}{c}F=0,800 \\
\text { Sig. }=0,527\end{array}$ & - & - & - & - & - & - \\
\hline CT4 & $\begin{array}{c}F=2,768 \\
\text { Sig. }=0,030\end{array}$ & $\begin{array}{c}F=2,075 \\
\text { Sig. }=0,087\end{array}$ & - & - & - & $\begin{array}{c}\text { HSD } \\
\text { Tukey }\end{array}$ & - & - \\
\hline IU1 & $\begin{array}{c}F=0,671 \\
\text { Sig. }=0,613\end{array}$ & $\begin{array}{c}F=0,261 \\
\text { Sig. }=0,903\end{array}$ & - & - & - & - & - & - \\
\hline IU2 & $\begin{array}{c}F=0,596 \\
\text { Sig. }=0,666\end{array}$ & $\begin{array}{c}F=1,804 \\
\text { Sig. }=0,131\end{array}$ & - & - & - & - & - & - \\
\hline
\end{tabular}

Fuente: Elaboración propia.

Finalmente, aplicamos la técnica estadística ANOVA, utilizada en pruebas paramétricas de hipótesis causales cuando existen más de dos grupos. En nuestro caso, el número de empleados se clasifica en menos de diez trabajadores, de once a veinticinco, de veintiséis a 100, de 100 a 250 y más de 250 trabajadores. Como se puede ver en la Tabla 8, los resultados de la prueba ANOVA sólo muestra diferencias significativas en AHS1, AHS2, CT1 y CT4, pero al aplicar los correspondientes Tests post hoc, sólo es posible encontrar pares con diferencias significativamente estadísticas en el caso CT1 ("es difícil de comprender lo que hace el sistema de cloud computing"). En este caso concreto, las empresas con más de 250 trabajadores presentan una media significativamente superior a las de diez a veinticinco trabajadores.

\section{Discusión, conclusiones e implicaciones}

La complejidad tecnológica derivada de la adopción del cloud se asocia negativamente con la intención de uso en función de la categoría laboral del encuestado, es decir si es directivo percibe la tecnología con mayor complejidad y con menor intención de adoptarla. Este resultado no ha resultado estadísticamente significativo, pero coincide con las conclusiones obtenidas por Tsai, Lee y Wu (2010) y, dentro del ámbito específico del cloud, por el estudio de Oliveira, Thomas y Espadanal (2014). Ello implica que, aunque los sistemas cloud son percibidos como útiles y fáciles de utilizar por las organizaciones, su implantación puede suponer ciertos retos tecnológicos que algunas empresas no pueden afrontar, como, por 
ejemplo, la necesidad de especialistas en TIC o las exigencias técnicas para proteger procesos y datos. Es decir, la complejidad actúa como un inhibidor de la intención de uso del cloud (Martins, Oliveira \& Thomas, 2016).

Estos resultados contrastan con el informe "Retos y Oportunidades del Cloud computing" (ONTSI, 2012), el cual analizó la situación e impacto del cloud computing en España, identificando oportunidades de crecimiento y estrategias de adopción de este tipo de modelo tecnológico, con especial atención en la pyme española.

Analizando el informe cabe destacar que el $45,2 \%$ de las pymes españolas con web conoce el cloud computing: el 20,5\% reconoce tener un sólido conocimiento de las soluciones cloud computing y su aplicabilidad en la empresa y un $24,7 \%$ ha "oído hablar" sobre la tecnología, conociendo algunos ejemplos, pero no la conoce en detalle. Por el contrario, un $54,9 \%$ de las pymes señala no conocer en absoluto la tecnología. Tres cuartas partes (77,5\%) de las empresas son conocedoras de la existencia del cloud, pero no han utilizado nunca soluciones basadas en cloud computing.

Los resultados obtenidos en nuestra investigación respecto al tamaño de las organizaciones corroboran este estudio y ponen de manifiesto este desconocimiento y como la influencia del tamaño de la organización (número de empleados) influye en la Complejidad Tecnológica, de modo que a mayor número de empleados, la complejidad de la tecnología cloud aumenta. Las empresas micropymes y pymes, que tengan menos de diez y 250 trabajadores respectivamente, perciben con menor dificultad de comprender lo que hace el sistema de cloud computing que aquellas empresas con más de 250 trabajadores que presentan una media significativamente superior.

Las valoraciones de los no directivos muestran una menor percepción de complejidad sobre los sistemas cloud que las de los directivos, para los cuales usar el cloud supone más tiempo y esfuerzo de aprendizaje y una mayor complejidad de uso.

Finalmente, por lo que respecta a la variable categoría laboral o puesto desempeñado por el entrevistado, conviene recordar que, en todas las organizaciones encuestadas, el cuestionario fue respondido por la persona con mayor conocimiento del proceso de adopción del cloud dentro de la misma o directamente por la dirección. Para ellos la cuestión sobre si es difícil de comprender lo que hace el sistema de cloud computing reflejó en el análisis comparativo que los directivos perciben una mayor complejidad tecnológica en los sistemas cloud, en comparación con los técnicos no directivos, constatando la mayor necesidad que la dirección y los cuadros medios tienen de tiempo y esfuerzo de aprendizaje en comparación con el personal técnico. Este hecho es explicable por las tareas propias del directivo, más ajenas, por así decirlo, a la frecuencia de uso de la tecnología.

Entre las limitaciones del estudio han estado que este se ha llevado a cabo a partir de empresas de Andalucía. Por lo tanto, hay que tener la correspondiente cautela a la hora de extrapolar los resultados a otros países o regiones, especialmente por el despliegue actual de las redes de alta velocidad, sus precios, por el número de microempresas existentes y, en consecuencia, su PIB regional. Así entre las futuras líneas de investigación que se sugieren estaría poder ampliar el estudio, incluyendo en la muestra un mayor número de empresas y organizaciones de otros ámbitos territoriales, incluyendo distintos países, como por ejemplo Portugal, diferenciando las etapas de desarrollo con respecto a la tecnología cloud y el contexto cultural o tecnológico. 


\section{Referencias}

Armbrust, M., Fox, A., Griffith, R., Joseph, A.D., Katz, R., Konwinski, A., ... Zaharia, M. (2010). A view of cloud computing. Communications of the ACM, 53 (4), 50-58. doi: 10.1145/1721654.1721672

Buyya, R., Yeo, C.S., Venugopal, S., Broberg, J. \& Brandic, I. (2009). Cloud computing and emerging IT platforms: Vision, hype, and reality for delivering computing as the $5^{\text {th }}$ utility. Future Generation Computer Systems, 25 (6), 599-616. doi: 10.1016/j.future.2008.12.001

Chou, D.C. \& Chou, A.Y. (2007). Analysis of a new information systems outsourcing practice: Softwareas-a-service business model. International Journal of Information Systems and Change Management, 2 (4), 392-405. doi: 10.1504/IJISCM.2007.017385

Forrester Research (2009). The global recessions will slow IT purchases growth to a crawl. (Global IT Market $\quad 12$ Report January 2009). Disponible en https://www.forrester.com/Global+IT+Market+Outlook+2009/fulltext/-/E-RES46676

Frost \& Sullivan (2008). Market demands for hosted UC services. Mountain View, California: Frost y Sullivan.

Goode, S., Lin, C., Tsai, J.C. \& Jiang, J.J. (2015). Rethinking the role of security in client satisfaction with Software-as-a-Service (SaaS) providers. Decision Support Systems, 70, 73-85. doi: 10.1016/j.dss.2014.12.005

Heiser, J. \& Nicolett, M. (2008). Assessing the security risks of cloud computing. (Report no. G00157782). Stamford, Connecticut: Gartner.

Hsu, J.S.-C., Shih, S.-P., Chiang, J.C. \& Liu, J.Y.-C. (2012). The impact of transactive memory systems on IS development teams' coordination, communication, and performance. International Journal of Project Management, 30 (3), 329-340. doi: 10.1016/j.ijproman.2011.08.003

Leavitt, N. (2009). Is cloud computing really ready for prime time? IEEE Computer Society, 42 (1), 15-20. doi: 10.1109/MC.2009.20

Lin, A. \& Chen, N.-C. (2012). Cloud computing as an innovation: Perception, attitude, and adoption. International Journal of Information Management, 32 (6), 533-540. doi: 10.1016/j.ijinfomgt.2012.04.001

Martins, R., Oliveira, T. \& Thomas, M.A. (2016). An empirical analysis to assess the determinants of SaaS diffusion in firms. Computers in Human Behavior, 62, 19-33. doi: 10.1016/j.chb.2016.03.049

Mathur, P. \& Nishchal, N. (2010). Cloud computing: New challenge to the entire computer industry. $1^{\text {st }}$ International Conference Parallel Distributed and Grid Computing (PDGC), 223-228. doi: 10.1109/PDGC.2010.5679897

National Institute of Standards and Technology (2011). Cloud Computing. Disponible en https://www.nist.gov/itl/cloud-computing

Observatorio Nacional de las Telecomunicaciones y de la Sociedad de la Información (2012). Cloud Computing. Retos Y Oportunidades. Madrid: Ministerio de Industria.

Oliveira, T., Thomas, M.A. \& Espadanal, M. (2014). Assessing the determinants of cloud computing adoption: An analysis of the manufacturing and services sectors. Information \& Management, 51 (5), 497-510. doi: 10.1016/j.im.2014.03.006

Pearlson, K.E. \& Saunders, C.S. (2009). Strategic management of information systems ( $4^{\text {th }}$ edition). New Jersey: Wiley.

Premkumar, G. \& Roberts, M. (1999). Adoption of new information technologies in rural small businesses. Omega, 27 (4), 467-484. doi: 10.1016/S0305-0483(98)00071-1

Red.es. (2011). Estudio sobre el impacto del Cloud Computing en el canal de distribución de software en España. Madrid: Ministerio de Industria, Energía y Turismo.

Rogers, E.M. (1995). Diffusion of Innovations ( $4^{\text {th }}$ edition). New York: Free Press.

Rogers, E.M. (2003). Diffusion of Innovations ( $5^{\text {th }}$ edition). New York: Free Press.

Schillewaert, N., Ahearne, M.J., Frambach, R.T. \& Moenaert, R.K. (2005). The adoption of information technology in the sales force. Industrial Marketing Management, 34 (4), 323-336. doi: 10.1016/j.indmarman.2004.09.013

Shah, M.H. \& Siddiqui., F.A. (2006). Organisational critical success factors in adoption of e-banking at the Woolwich bank. International Journal of Information Management, 26 (6), 442-456. doi: 10.1016/j.ijinfomgt.2006.08.003

Sosinski, B. (2011). ¿Qué es la nube? El futuro de los sistemas de información. Madrid: ANAYA.

Taylor, S. \& Todd, P.A. (1995). Understanding information technology usage: A test of competing models. Information Systems Research, 6 (2), 144-176. doi: 10.1287/isre.6.2.144

Tsai, M.-C., Lee, W. \& Wu, H.-C (2010). Determinants of RFID adoption intention: Evidence from Taiwanese retail chains. Information \& Management, 47 (5-6), 255-261. doi: 
10.1016/j.im.2010.05.001

Wang, C., Chow, S.S.M., Wang, Q., Ren, K. \& Lou, W. (2013). Privacy-preserving public auditing for secure cloud storage. IEEE Transactions on Computers, 62 (2), 362-375. doi: 10.1109/TC.2011.245

Xin, M. \& Levina, N. (2008). Software-as-a-service model: Elaborating client-side adoption factors. In R. Boland, M. Limayem \& B. Pentland (Eds.), Proceedings of the $29^{\text {th }}$ International Conference on Information Systems (pp. 14-17). doi: 10.2139/ssrn.1319488

Pedro R. Palos es profesor asociado del Departamento de Dirección de Empresa de la Universidad de Extremadura. Lleva más de 20 años trabajando en Empresas del sector de Tecnologías de la Información en diferentes puestos directivos. Ha sido miembro de los comités ejecutivos de la Asociación Española de Empresas e-Learning-CEOE, la Confederación de Empresas de Comercio y Servicios de Sevilla, Secretario del Colegio Profesional de Ingenieros Técnicos en Informática de Andalucía y Secretario General de la Federación de Empresas de Informática de Andalucía (FIA). Participa con distintas ponencias y artículos en diversos congresos y programas sobre economía digital, emprendimiento y management tanto nacional como internacional, siendo sus líneas de investigación principales Marketing Digital y Sistemas de Información. Es miembro del Grupo de Investigación MARKETUR Research Group de la Universidad de Extremadura. Dirección institucional: Facultad de Empresa, Finanzas y Turismo de la Universidad de Extremadura, Av. Universidad, s/n. 10003, Cáceres, España.

MARISOl B. CorreiA es profesora en la Escola Superior de Gestão, Hotelaria e Turismo de la Universidad del Algarve y es miembro colaborador del Centro de Estudos em Gestão (CEG-IST) de la Universidad de Lisboa. Desenvuelve actividades de investigación en Tecnologías de Información y Comunicación aplicadas al Turismo, Administración, Hospitalidad y Marketing, Cloud Computing, Social Media, Evaluación de websites, Business intelligence y Computación Evolutiva. Es autora de varios artículos en revistas y conferencias internacionales y ha participado en varios proyectos nacionales. Dirección institucional: Escola Superior de Gestão, Hotelaria e Turismo, Universidade do Algarve, Campus da Penha, 8005-139 Faro, Portugal.

Submetido em 14 junho 2016

Aceite em 28 julho 2016 